\title{
Fast Frictionless Expansion of an Optical Lattice
}

\author{
C. Yuce \\ Physics Department, Anadolu University, Eskisehir, Turkey
}

(Dated: November 5, 2018)

\begin{abstract}
We investigate fast frictionless expansion of an optical lattice with dynamically variable spacing (accordion lattice). We design an expansion trajectory that yields a final state identical to the initial state up to an irrelevant phase factor. We discuss the effect of additional force and nonlinear interaction on the fast frictionless expansion.
\end{abstract}

\section{INTRODUCTION}

In an adiabatic process, an eigenstate of a time dependent Hamiltonian follows its instantaneous eigenstate. However, adiabatic processes typically need timescales longer than lifetimes or coherence times of the system, therefore hindering their applicability. This has motivated researchers to find a way to speed up the process to reach the same instantaneous adiabatic eigenstate [1]. Recently, a new technique has been introduced 2 26 and experimentally realized with ultracold ${ }^{87} R b$ atoms in a harmonic trap [11, 12]. The technique is based on engineering the time dependent parameters of the Hamiltonian. The fast schemes to get the same final state as the one after adiabatic process is called shortcut to adiabaticity [6] or superadiabatic [25]. It is known that fast schemes are not adiabatic in between initial and final times. In the experiment [12], the trap is decompressed by a factor of 15 within $35 \mathrm{~ms}$, which is much less than typical adiabatic process of a few seconds. Choi et al. discussed that optimal cooling of an atomic species may be obtained by means of sympathetic cooling with another species whose trapping frequency is dynamically changed to maintain constancy of the Lewis-Riesenfeld adiabatic invariant [19]. Recently, nearly perfect fidelity for a twolevel quantum system was achieved experimentally [25]. The fast transitionless mechanism has also been extended to the Gross-Pitaevskii (GP) equation [26]. Most of the investigations so far have been based on ultracold atoms in a harmonic trap.

In this paper, we will explore the possibility of fast frictionless expansion for an optical lattice. We will show that a correct choice of expansion trajectory allows us to get a final adiabatic state in a non-adiabatic way. Our method of obtaining fast adiabatic transformation is to use a lattice with dynamically variable spacing (accordion lattice) $[27-30]$. A continuous change of the lattice periodicity from $0.96 \mu \mathrm{m}$ to $11.2 \mu \mathrm{m}$ in one second was achieved in an early experiment with an optical lattice 28]. Recent experiment with ultracold atoms of ${ }^{87} \mathrm{Rb}$ trapped in a two-dimensional optical lattice demonstrated the variation of the spacing of the lattice in two directions from $2.2 \mu \mathrm{m}$ to $5.5 \mu \mathrm{m}$ in a few milliseconds [30] using dual axis acousto-optic deflectors. Here, we will give the conditions for fast frictionless expansion for an accordion lattice. Such accordion lattices are useful since the final lattice spacing can be made large enough to be resolved in an experiment. Note that, in principle, the lattice spacing can be extended to any size smaller than the laser beam waist. This allows imaging of the atoms at individual sites.

\section{ACCORDION LATTICE}

In contrast to electrons in a crystal lattice, optical lattices allow us to change parameters, such as potential depth and lattice spacing. The potential depth of the optical lattice can be modulated by changing the power of the laser. The lattice spacing can be changed by changing the wavelength of the laser or by changing the relative angle between the two laser beams 27 30. Let us consider ultracold atoms in an optical lattice with variable spacing and potential depth. We assume that the transverse motion of the atoms is frozen (i.e., we are dealing with a 1-D problem). The description of a condensate in $1-\mathrm{D}$ is based on the Hamiltonian

$$
H=\frac{p^{2}}{2 m}+V(t) \cos \left(2 k_{L} \frac{x}{\Lambda(t)}\right)+\frac{m \omega^{2}(t)}{2} x^{2} .
$$

where $m$ is the atomic mass, $V(t)$ is the lattice depth, $k_{L}$ is the optical lattice wave number, $\omega(t)$ is the time dependent angular frequency and $\Lambda(t)$ is the scale parameter describing the expansion of the lattice. The initial and final lattice scalings are given by

$$
\Lambda(t=0)=1, \Lambda\left(t=t_{f}\right)=c,
$$

where the constant $c$ is the ratio of the final spacing to the initial spacing.

Initially only the periodic potential is present in the system. When the system starts to expand by changing the lattice spacing, the parabolic potential is also added. The combined presence of the periodic and parabolic potentials enables us to get fast frictionless expansion. Finally, the parabolic potential is turned off at $t=t_{f}$ such that

$$
\omega(t=0)=\omega\left(t=t_{f}\right)=0 .
$$

The system is initially in the stationary state. To find the time evolution of the state for the Hamiltonian (1), we introduce a transformation on the wave function as

$$
\Psi(x, t)=\exp \left(-i g x^{2} / 2+\frac{\hbar}{2 m} \int g d t\right) \Phi(x, t),
$$


with a subsequent transformation on the coordinate

$$
x=z \Lambda(t) .
$$

where $g(t)$ is a time dependent function to be determined later. Indeed, the scale transformation on the coordinate accounts for the expansion of the condensate. Now let us substitute these transformations into the corresponding Schrodinger equation for the Hamiltonian (11). Note that due to the scaling of coordinate, the time derivative operator transforms as $\partial / \partial t \rightarrow \partial / \partial t-(\dot{\Lambda} / \Lambda) z \partial / \partial z$, where dot denotes time derivation. Choosing

$$
g(t)=-\frac{m}{\hbar} \frac{\dot{\Lambda}}{\Lambda}
$$

yields

$$
i \hbar \frac{\partial \Psi}{\partial t}=-\frac{\hbar^{2}}{2 m \Lambda^{2}} \frac{\partial^{2} \Psi}{\partial z^{2}}+V \cos \left(2 k_{L} z\right) \Phi+\frac{m}{2} \Omega^{2} z^{2} \Phi
$$

where $\Omega^{2}$ is given by

$$
\Omega^{2}(t)=\Lambda^{2} \omega^{2}+\Lambda \frac{\partial^{2} \Lambda}{\partial t^{2}}
$$

To sum up, the effects of lattice spacing are equivalent to the effective time dependent mass and the effective parabolic potential in the stationary frame. The first term in the right hand side of (8) is due to the external one while the second one is induced due to the variation of the lattice spacing. Indeed, the last formula explains the difficulties of keeping atoms trapped in an experiment with an accordion lattice [29, 30]. In the absence of external harmonic trap, $\omega^{2}=0$, the system is left with the induced parabolic potential that depends on the acceleration, $\frac{\partial^{2} \Lambda}{\partial t^{2}}$. Since the expansion starts at $t=0$ and stops at $t=t_{f}$, the acceleration takes both positive and negative values in the interval $0<t<t_{f}$. Hence the repulsive potential with an imaginary frequency, $\Omega^{2}<0$, has the effect of pushing the atoms out of the condensate. To tackle the problem, the lattice depth was ramped from $h \times 1.5 \mathrm{~Hz}$ to $h \times 37 \mathrm{~Hz}$ in $5 \mathrm{~ms}$ in the experiment [30]. Here we instead suggest decreasing the lattice depth. The first reason for this is that the fast frictionless expansion can be achieved by engineering the potential depth as

$$
V(t)=\frac{V_{0}}{\Lambda^{2}(t)}
$$

Hence the potential depth decreases as the system expands. Secondly, we demand that no effective harmonic potential acts on the system, $\Omega^{2}=0$. In other words, we choose $\omega^{2}(t)$ in such a way that it cancels the effective parabolic potential so that there is only periodic potential in the system from $t=0$ to $t=t_{f}$. Hence, we choose

$$
\omega^{2}(t)=-\Lambda^{-1} \frac{\partial^{2} \Lambda}{\partial t^{2}}
$$

This choice of external frequency guarantees that ultracold atoms stay inside the optical lattice.

The conditions (910) reduce the equation (7) to the following one

$$
i \hbar \frac{\partial \Phi}{\partial \tau}=-\frac{\hbar^{2}}{2 m} \frac{\partial^{2} \Phi}{\partial z^{2}}+V_{0} \cos \left(2 k_{L} z\right) \Phi
$$

In the last step, we made another transformation on time $\tau=\int_{0}^{t} \Lambda^{-2}\left(t^{\prime}\right) d t^{\prime}$

It is interesting to observe that the Schrodinger equation for the Hamiltonian (11) can be transformed into the another Schrodinger equation (11) with the constant potential depth. We can view (11) as describing another condensate with a different time parameter.

We find a new Hamiltonian $H^{\prime}=\frac{p^{2}}{2 m}+V_{0} \cos \left(2 k_{L} z\right)$ for the system described by the Hamiltonian (1). The final state of $H^{\prime}$ at $t_{f}$ can be made identical up to a global phase factor to the final state of the adiabatic evolution with $H$ if we impose some conditions on the scale parameter $\Lambda(t)$. By virtue of (46), we conclude that they are given by

$$
\dot{\Lambda}(0)=\dot{\Lambda}\left(t_{f}\right)=0, \quad \ddot{\Lambda}(0)=\ddot{\Lambda}\left(t_{f}\right)=0,
$$

where dot is the time derivation with respect to the parameter $t$. The acceleration, $\ddot{\Lambda}$, is set to zero at initial and final times since the initial and final external frequencies are assumed to be zero (3/10). These conditions together with the conditions (2) guarantee the fast transitionless evolution.

Here we will mainly be interested in three different solutions for $\Lambda(t)$. The first one satisfying the relations (2[12) is the polynomial solution

$$
\Lambda_{1}(t)=(c-1)\left(\frac{1}{c-1}+10 \frac{t^{3}}{t_{f}^{3}}-15 \frac{t^{4}}{t_{f}^{4}}+6 \frac{t^{5}}{t_{f}^{5}}\right)
$$

and the second one is given by

$$
\Lambda_{2}(t)=(c-1)\left(\frac{1}{c-1}+\frac{t}{t_{f}}-\frac{1}{2 n \pi} \sin \left(\frac{2 n \pi t}{t_{f}}\right)\right) \text {. }
$$

where $n$ is a positive integer. For these trajectories, the external frequency can be calculated using (10). In Fig-1 and Fig-2, we plot $\Lambda$ and corresponding $\omega^{2}$. The dashed (solid) curve is for $\Lambda_{1}\left(\Lambda_{2}\right.$ with $\left.n=3\right)$. We assume the lattice is expanded by a factor of 2.5. As expected, the sign of $\omega^{2}(t)$ changes in time. There are some differences in implementing fast frictionless processes in harmonic traps and optical lattices. For a harmonically trapped system, we need the harmonic potential to achieve the expansion of the system. However, expansion for an optical lattice can be achieved either by changing the wavelength of the laser or the relative angle between the two laser beams. The external parabolic potential is necessary for an optical lattice to cancel the induced parabolic 


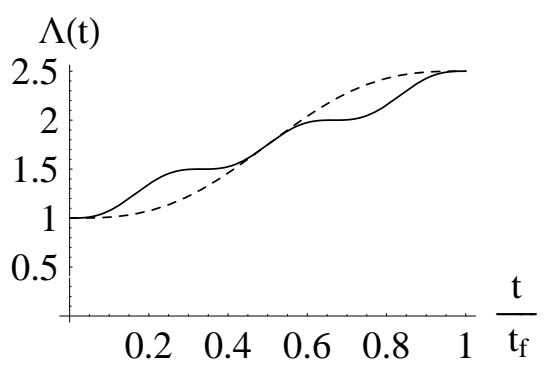

FIG. 1: $\Lambda(t)$ versus $t / t_{f}$ for $c=2.5$. The dashed (solid) curve is for $\Lambda_{1}(t)\left(\Lambda_{2}(t)\right.$ with $\left.n=3\right)$.

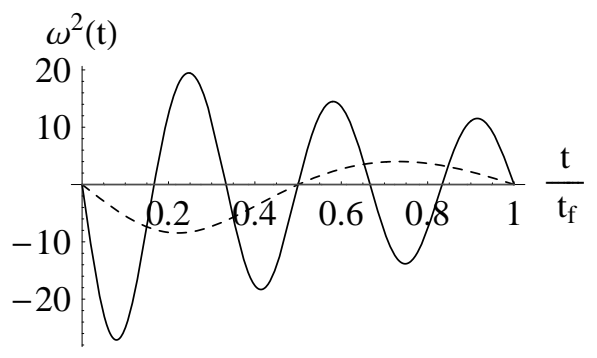

FIG. 2 : $\omega^{2}(t)$ versus $t / t_{f}$ for $c=2.5$. The dashed (solid) curve is for $\Lambda_{1}(t)\left(\Lambda_{2}(t)\right.$ with $\left.n=3\right)$.

potential, which pushes the atoms out of the condensate since it becomes expulsive for certain time intervals.

One interesting trajectory was already realized in the experiment [30], where the lattice was expanded by a factor of 2.5 in a few milliseconds

$$
\Lambda_{3}(t)=1+0.75\left(\operatorname{erf}\left\{7.14\left(\frac{t}{t_{f}}-0.5\right)\right\}+1\right) .
$$

where erf is the Gauss error function. $\Lambda_{3}(t)$ satisfies (12) to a very good approximation. Fig-3 and Fig-4 plot $\Lambda_{3}(t)$ and corresponding $\omega^{2}(t)$. As can be seen, $\omega^{2}$ is negative between $0<t<t_{f} / 2$, while it is positive otherwise. We propose to repeat the experiment [28] with the same trajectory but a different potential depth and a different frequency. If they change according to (910), then fast frictionless expansion can be realized.

So far, we have studied 1-D optical lattice with variable spacing. The generalization to higher dimensions is straightforward. In this section, we will discuss the effects of additional force, harmonic potential and scattering length on the fast frictionless expansion. Let us first consider the presence of an additional force, $F(t)$, in the Hamiltonian (11). The force can be a constant gravitational force or a periodically changing one. The latter is experimentally realized by generating periodical frequency shift between the two laser beams [31]. The dynamical responses of the particle to the constant force and periodically changing force are the well-known phenomena of Bloch oscillations [32-34] and dynamical localization [35], respectively. The additional force, $F(t)$ transforms as $F(\tau) \Lambda^{3}(\tau)$ in the equation (11). Hence,

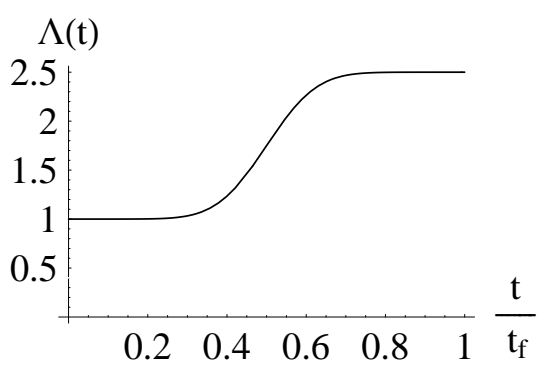

FIG. 3: $\Lambda_{3}(t)$ versus $t / t_{f}$. The lattice is expanded by a factor of 2.5 from $2.2 \mu \mathrm{m}$ to $5.5 \mu \mathrm{m}$ in a few milliseconds.

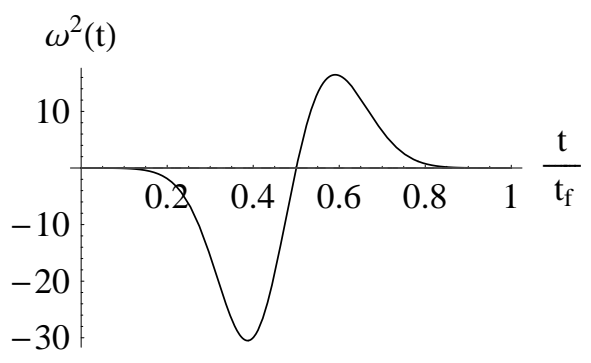

FIG. 4: $\omega^{2}(t)$ versus $t / t_{f}$ for $\Lambda_{3}(t)$

the picture for Bloch oscillation and dynamical localization change a lot for the trajectories (13)14[15). The fast frictionless expansion without destroying the Bloch oscillation and dynamical localization would be possible if the force transforms as $F / \Lambda^{3}$.

Suppose now that the system is initially prepared in the combined presence of the periodic plus parabolic potentials. Hence, the initial and final frequencies are given by $\omega(0)=\omega\left(t_{f}\right)=\omega_{0}$. In this case, the condition (12) is still valid but (10) is not. The fast frictionless expansion for this case is possible if $\Omega^{2}$ is given by $\Omega^{2}=\omega_{0}^{2} / \Lambda^{2}$ in the equation (7). The specific external frequency, $\omega(t)$, satisfying this condition can be found easily using the equation (8). Let us now discuss dipole oscillation. In the experiment for 3-D BEC [36], the trap in the presence of the optical lattice was suddenly shifted. It was observed that the subsequent oscillations of the BEC are undamped if the initial displacement is small, but become dissipative if the initial displacement exceeds a critical value. Fertig et al. 38] induced dipole oscillations of 1-D Bose gases for different values of the axial lattice depth. They observed a transition from underdamping to overdamping with increasing lattice depth. Due to strong quantum fluctuations, mean-field theories fail for the description of the system. Consider an experiment like the ones in [36, 37] to study dipole oscillation for an accordion lattice. The amplitude of the small dipole oscillation increases with $\Lambda$ without leading instability since the lattice is expanding (5). The fast frictionless expansion preserves dipole oscillation.

Let us now discuss the extension of the fast frictionless expansion to GP equation. If the scattering length is 
changed according to $a_{s}(t)=a_{s}(0) / \Lambda^{2}$ via Feshbach resonance, then adiabatic final state can be obtained nonadiabatically. As a result, we say that the fast frictionless expansion is not limited to noninteracting case.

In conclusion, we have shown that the non-adiabatic expansion of an optical lattice in few milliseconds may lead to adiabatic final states provided that the expansion trajectory satisfies (12) and the external parabolic potential is applied to the system. This prediction can be tested through experiments similar to the one described in [30]. Since the non-adiabatic protocol preserves the initial state after expansion, it allows straightforward imaging of the optical lattice. We have also discussed the effect of additional forces and harmonic trapping. Finally, we argue that fast frictionless expansion is possible even in the presence of nonlinear interaction provided that the scattering length is decreased proportionally to $\Lambda^{2}$ via Feshbach resonance.
* Electronic address: cyuce@anadolu.edu.tr

[1] P. Salamon, K. H. Hoffmann, Y. Rezek, and R. Kosloff, Phys. Chem. Chem. Phys. 11, 1027 (2009).

[2] Xi Chen, E. Torrontegui, Dionisis Stefanatos, Jr-Shin Li, J. G. Muga, Phys. Rev. A 84, 043415 (2011).

[3] S. Ibáñez, S. Mart nez-Garaot, Xi Chen, E. Torrontegui, J. G. Muga, Phys. Rev. A 84, 023415 (2011).

[4] Xi Chen, E. Torrontegui, J. G. Muga, Phys. Rev. A 83, 062116 (2011).

[5] E. Torrontegui, S. Ibanez, Xi Chen, A. Ruschhaupt, D. Guery-Odelin, J. G. Muga, Phys. Rev. A 83, 013415 (2011).

[6] Xi Chen, I. Lizuain, A. Ruschhaupt, D. Guery-Odelin, J. G. Muga, Phys. Rev. Lett. 105, 123003 (2010).

[7] X. Chen and J. G. Muga, Phys. Rev. A 82, 053403 (2010).

[8] X. Chen, A. Ruschhaupt, S. Schmidt, A. del Campo, D. Guery-Odelin, and J. G. Muga, Phys. Rev. Lett. 104, 063002 (2010).

[9] J. G. Muga, X. Chen, S. Ibanez, I. Lizuain, and A. Ruschhaupt, J. Phys. B 43, 085509 (2010).

[10] S. Masuda and K. Nakamura, Proc. R. Soc. A 466, 1135 (2010).

[11] J. F. Schaff, X. L. Song, P. Vignolo, and G. Labeyrie, Phys. Rev. A 82, 033430 (2010).

[12] J. F. Schaff, X. L. Song, P. Capuzzi, P. Vignolo, and G. Labeyrie, Europhys. Lett. 93, 23001 (2011).

[13] D. Stefanatos, J. Ruths, and Jr-Shin Li, Phys.Rev. A 82, $063422(2010)$.

[14] S. Masuda and K. Nakamura, Physical Review A 84, 043434 (2011).

[15] J. F. Schaff, P. Capuzzi, G. Labeyrie and P. Vignolo, New J. Phys. 13113017 (2011).

[16] B. Andresen, K. H. Hoffmann, J. Nulton, A. Tsirlin, and P. Salamon, Eur. J. Phys. 32, 827 (2011).

[17] Y. Li, L.-A. Wu, and Z.-D. Wang, Phys. Rev. A 83, 043804 (2011).

[18] A. del Campo, Phys. Rev. A 84, 031606(R) (2011).
[19] S. Choi, R. Onofrio, and B. Sundaram, Phys. Rev. A 84 051601(R) (2011).

[20] M. Fasihi, Y. Wan, M. Nakahara, J. Phys. Soc. Jpn. 81, 024007 (2012).

[21] B. Andresen, K. H. Hoffmann, J. Nulton, A. Tsirlin and P. Salamon, Eur. J. Phys. 32827 (2011).

[22] M. V. Berry, J. Phys. A 42, 365303 (2009).

[23] C Yuce, A Kilic and A Coruh, Phys. Scr. 74114 (2006).

[24] K. H. Hoffmann, et. al, EPL 9660015 (2011).

[25] Mark G. Bason, et. al, Nature Phys. 8, 147 (2012).

[26] J. G. Muga, X. Chen, A. Ruschhaupt, and D. GuéryOdelin, J. Phys. B 42, 241001 (2009).

[27] Leonardo Fallani, Chiara Fort, Jessica Lye, Massimo Inguscio, Opt. Express 13, 4303 (2005).

[28] T. C. Li, H. Kelkar, D. Medellin, M. G. Raizen, Opt. Express 16, 5465 (2008).

[29] R. A. Williams, J. D. Pillet, S. Al-Assam, B. Fletcher, M. Shotter, C. J. Foot, Optics Express, 21, 16977 (2008).

[30] S. Al-Assam, R. A. Williams, C. J. Foot, Phys. Rev. A 82, 021604(R) (2010).

[31] H. Lignier, C. Sias, D. Ciampini, Y. Singh, A. Zenesini, O. Morsch, E. Arimondo, Phys. Rev. Lett. 99, 220403 (2007).

[32] M. Ben Dahan, E. Peik, J. Reichel, Y. Castin, and C. Salomon, Phys. Rev. Lett. 76, 4508 (1996).

[33] O. Morsch, J. H. Muller, M. Cristiani, D. Ciampini, and E. Arimondo, Phys. Rev. Lett. 87, 140402 (2001).

[34] B. P. Anderson and M. Kasevich, Science 282, 1686 (1998).

[35] D. H. Dunlap and V. M. Kenkre, Phys. Rev. B 34, 3625 (1986).

[36] S. Burger, F. S. Cataliotti, C. Fort, F. Minardi, M. Inguscio, M. L. Chiofalo, M. P. Tosi, Phys. Rev. Lett. 86, 4447 (2001).

[37] A. Smerzi, A. Trombettoni, P.G. Kevrekidis, A.R. Bishop, Phys. Rev. Lett. 89, 170402 (2002).

[38] C. D. Fertig et al., Phys. Rev. Lett. 94, 120403 (2005). 